\title{
Collapse and revival of excitations in Bose-Einstein condensates
}

\author{
M. Möttönen, S. M. M. Virtanen and M. M. Salomaa \\ Materials Physics Laboratory, Helsinki University of Technology \\ P. O. Box 2200 (Technical Physics), FIN-02015 HUT, Finland
}

(Dated: November 5, 2018)

\begin{abstract}
We study the energies and decay of elementary excitations in weakly interacting Bose-Einstein condensates within a finite temperature gapless second order theory. The energy shifts for the high-lying collective modes turn out to be systematically negative compared with the HartreeFock-Bogoliubov-Popov approximation and the decay of the low-lying modes are found to exhibit collapse and revival effects. In addition, perturbation theory is used to qualitatively explain the experimentally observed Beliaev decay process of the scissors mode.
\end{abstract}

\section{INTRODUCTION}

The partially Bose-Einstein condensed trapped atomic gases provide an excellent testbench for developing finite temperature quantum theories. These weakly interacting systems can be modelled from first principles, and the experiments yield accurate and detailed information for comparison. Especially, the energies and decay rates of low-energy collective excitations have been measured at different temperatures and the results provide stringent tests for theoretical models.

For dilute condensates at temperatures much lower than the condensation temperature $T_{\mathrm{c}}$, the Bogoliubov approximation consisting of the Gross-Pitaevskii (GP) equation for the condensate wave function and the Bogoliubov equations for the quasiparticle excitations has proven to be accurate in describing the collective modes of the system. For higher temperatures one has to take into account the effects of the thermal gas component. Developing a theory that is computationally feasible and correctly models the system at temperatures approaching $T_{\mathrm{c}}$ is a challenging task. The most commonly used finite-temperature theory is the HartreeFock-Bogoliubov-Popov (HFB-Popov) approximation. It neglects the dynamics of the thermal gas and the modifications in particle correlations induced by the condensate, but predicts quasiparticle energies in fair agreement with the experiments [1]. The energy of the quadrupole modes having azimuthal angular momentum quantum numbers $q_{\theta}= \pm 2$ deviates from the theoretical prediction for temperatures above $0.6 T_{\mathrm{c}}$, but lately this deviation has been interpreted to mainly arise from improper modelling of the time-dependent external potential used in the experiments to excite the collective modes [2].

In order to take into account the leading order quasiparticle interactions and the correlations induced by the condensate in the inhomogeneous case, several theoretical approaches have been suggested [3-10]. The dynamics of the condensate and the thermal gas has also been studied using various kinetic theories [11-15]. The second order theory for inhomogeneous, partially condensed gases presented in Refs. $[9,10]$ uses systematic perturbation theory to take into account the interaction terms in the Hamiltonian. Recently, this theory was extended to take into account the time-dependent external perturbation used to drive the system in the experiments, leading to an agreement with the measured energies and the damping rates of the collective modes $[2,16]$.

The second order theories are computationally challenging, and there has been only a few numerical investigations of their predictions $[2,17,18]$. In this paper, we calculate the spectral distributions of the quasiparticle energies for a partially condensed Bose-Einstein condensate (BEC), and compare the quasiparticle energies to the HFB-Popov results as functions of temperature. Especially, we analyze the quasiparticle dynamics implied by the spectral distributions, observing that some collective modes should exhibit notable collapse and revival effects in trapped condensates. The possible existence of this phenomenon has been pointed out previously in Refs. [9, 10] (see also Ref. [19]), but, however, it has not been studied in detail before. The collapse and revival of the excitations indicates that the energies and the damping rates alone do not suffice to describe the dynamics of these modes, i.e., the commonly used damped sinusoidal fit to the experimental data may not be sufficient to describe the longer term dynamics of some modes.

The structure of the paper is the following: In Section II, we describe the second order theory used in the analysis. Section III is devoted to a discussion of the numerical methods used to calculate the excitation spectra and the dynamics of modes. In Section IV, we analyze the second order corrections to the excitation spectrum as functions of temperature, and in Section $\mathrm{V}$ we study the decay of certain modes. Section VI consists of discussion and summary of the results.

\section{SECOND ORDER THEORY}

In this section, following Refs. $[9,10]$, we present the second order formalism for calculating the quasiparticle spectral distributions for a partially condensed, dilute, trapped BEC at finite temperatures. The starting point is the usual second quantized Hamiltonian for structureless bosons

$$
\hat{H}=\sum_{i j}\left\langle i\left|\hat{H}^{\mathrm{sp}}\right| j\right\rangle \hat{a}_{i}^{\dagger} \hat{a}_{j}+\frac{1}{2} \sum_{i j k m}\langle i j|V| k m\rangle \hat{a}_{i}^{\dagger} \hat{a}_{j}^{\dagger} \hat{a}_{k} \hat{a}_{m},
$$


where the creation and annihilation operators for a particle in state $|i\rangle$ are denoted by $\hat{a}_{i}^{\dagger}$ and $\hat{a}_{i}$, respectively. The single particle Hamiltonian is given by the sum of the kinetic energy and the external trapping potential as

$$
\hat{H}^{\mathrm{sp}}=-\frac{\hbar^{2} \nabla^{2}}{2 m}+V_{\mathrm{trap}}(\mathbf{r}),
$$

and the dominant $s$-wave scattering at low temperatures can be modelled by the effective low energy interaction potential

$$
V(\mathbf{r})=\frac{4 \pi a \hbar^{2} \delta(\mathbf{r})}{m},
$$

where $a$ is the scattering length and $m$ the atomic mass. This effective potential is inapplicable at high energies and leads to ultraviolet divergences in the theory which have to be renormalized in a proper way, see Appendix.

We choose to use a canonical ensemble with fixed total number of particles $N$. By defining the bosonic number conserving operators $\hat{\alpha}_{i}=\left[\left(\hat{N}_{0}+1\right)^{-1 / 2} \hat{a}_{0}\right]^{\dagger} \hat{a}_{i}$, where the index 0 refers to the condensate state and $\hat{N}_{0}=\hat{a}_{0}^{\dagger} \hat{a}_{0}$, one can write the Hamiltonian (1) as

$$
\hat{H}=\sum_{i=0}^{4} \hat{H}_{i}+O\left(N_{0}\left[\hat{\delta} / N_{0}\right]^{5 / 2}\right),
$$

where

$$
\begin{aligned}
\hat{H}_{0}= & N_{0}\left[\left\langle 0\left|\hat{H}^{\mathrm{sp}}\right| 0\right\rangle+\frac{1}{2} N_{0}\left\langle 00\left|V^{\mathrm{s}}\right| 00\right\rangle\right] \\
\hat{H}_{1}= & \sqrt{N_{0}} \sum_{i \neq 0}\left[\left\langle i\left|\hat{H}^{\mathrm{sp}}\right| 0\right\rangle+N_{0}\left\langle i 0\left|V^{\mathrm{s}}\right| 00\right\rangle\right] \hat{\alpha}_{i}^{\dagger}+\text { h.c. }, \\
\hat{H}_{2}= & \sum_{i j \neq 0}\left[\left\langle i\left|\hat{H}^{\mathrm{sp}}\right| j\right\rangle-\lambda \delta_{i j}+2 N_{0}\left\langle 0 i\left|V^{\mathrm{s}}\right| j 0\right\rangle\right] \hat{\alpha}_{i}^{\dagger} \hat{\alpha}_{j} \\
& +\sum_{i j \neq 0}\left[\frac{N_{0}}{2}\left\langle i j\left|V^{\mathrm{s}}\right| 00\right\rangle \hat{\alpha}_{i}^{\dagger} \hat{\alpha}_{j}^{\dagger}+\text { h.c. }\right]+\lambda\left\langle\hat{N}_{\mathrm{ex}}\right\rangle, \\
\hat{H}_{3}= & \sum_{i j k \neq 0}\left[\sqrt{N_{0}}\left\langle i j\left|V^{\mathrm{s}}\right| k 0\right\rangle \hat{\alpha}_{i}^{\dagger} \hat{\alpha}_{j}^{\dagger} \hat{\alpha}_{k}+\text { h.c. }\right], \\
\hat{H}_{4}= & \sum_{i j k m \neq 0} \frac{1}{2}\left\langle i j\left|V^{\mathrm{s}}\right| k m\right\rangle \hat{\alpha}_{i}^{\dagger} \hat{\alpha}_{j}^{\dagger} \hat{\alpha}_{k} \hat{\alpha}_{m},
\end{aligned}
$$

and $\hat{\delta}=\hat{N}_{\text {ex }}-\left\langle\hat{N}_{\text {ex }}\right\rangle$ is the number fluctuation operator of the noncondensate particles. The symmetrized matrix elements of the two-particle interaction potential $V(\mathbf{r})$ are defined as

$$
\left\langle i j\left|V^{\mathrm{s}}\right| k m\right\rangle=\frac{1}{2}[\langle i j|V| k m\rangle+\langle j i|V| k m\rangle],
$$

and $\lambda$ as

$$
\lambda=\left\langle 0\left|\hat{H}^{\mathrm{sp}}\right| 0\right\rangle+N_{0}\left\langle 00\left|V^{\mathrm{s}}\right| 00\right\rangle,
$$

where the average number of atoms in the condensate state is given by $N_{0}=N-\left\langle\hat{N}_{\text {ex }}\right\rangle$. Above the averages $\langle\ldots\rangle$ refer to quantum expectation values and h.c. stands for hermitian conjugate.

In the zeroth order approximation, one solves the ground state $|0\rangle$ of $\hat{H}_{0}$ alone, which makes the linear Hamiltonian $\hat{H}_{1}$ to vanish. The excitations are found in lowest order by diagonalizing $\hat{H}_{2}$ and the number of the condensed particles $N_{0}$ has to be tuned such that the total number of particles satisfies $N=N_{0}+N_{\text {ex }}$.

It is convenient the use an orthonormal single-particle basis $\zeta_{i}(\mathbf{r})=\langle\mathbf{r} \mid i\rangle$ for all $i=0,1, \ldots$, where $\zeta_{0}(\mathbf{r})$ is the condensate wave function given by the Gross-Pitaevskii equation

$$
-\frac{\hbar^{2}}{2 m} \nabla^{2} \zeta_{0}+V_{\text {trap }}(\mathbf{r}) \zeta_{0}+N_{0} U_{0}\left|\zeta_{0}(\mathbf{r})\right|^{2} \zeta_{0}=\lambda \zeta_{0}(\mathbf{r}),
$$

with $U_{0}=4 \pi a \hbar^{2} / m$. The GP equation is obtained by minimizing $\left\langle\hat{H}_{0}\right\rangle$ with respect to $\zeta_{0}(\mathbf{r})$. Diagonalizing $\hat{H}_{2}$ using the Bogoliubov transformation $\hat{\beta}_{i}=$ $\sum_{j \neq 0}\left[U U_{i j}^{*} \hat{\alpha}_{j}-V_{i j}^{*} \hat{\alpha}_{j}^{\dagger}\right]$ results in the Bogoliubov equations

$$
\left(\begin{array}{cc}
\mathcal{L}(\mathbf{r}) & \mathcal{M}(\mathbf{r}) \\
-\mathcal{M}^{*}(\mathbf{r}) & -\mathcal{L}(\mathbf{r})
\end{array}\right)\left(\begin{array}{c}
u_{i}(\mathbf{r}) \\
v_{i}(\mathbf{r})
\end{array}\right)=\epsilon_{i}\left(\begin{array}{c}
u_{i}(\mathbf{r}) \\
v_{i}(\mathbf{r})
\end{array}\right),
$$

where $u_{i}(\mathbf{r})=\sum_{j \neq 0} U_{i j} \zeta_{j}(\mathbf{r})$ and $v_{i}(\mathbf{r})=\sum_{j \neq 0} V_{i j} \zeta_{j}^{*}(\mathbf{r})$ are the quasiparticle amplitudes, $\epsilon_{i}$ the quasiparticle energies, and operators $\mathcal{L}(\mathbf{r})=\hat{H}^{\mathrm{sp}}-\lambda+2 N_{0} U_{0}\left|\zeta_{0}(\mathbf{r})\right|^{2}$ and $\mathcal{M}(\mathbf{r})=N_{0} U_{0} \zeta_{0}^{2}(\mathbf{r})$ have been introduced. The quasiparticle amplitudes must satisfy the orthogonality and symmetry relations

$$
\begin{aligned}
& \int \mathrm{d} \mathbf{r}\left[u_{i}(\mathbf{r}) u_{j}^{*}(\mathbf{r})-v_{i}(r) v_{j}^{*}(\mathbf{r})\right]=\delta_{i j}, \\
& \int \mathrm{d} \mathbf{r}\left[u_{i}(\mathbf{r}) v_{j}(\mathbf{r})-v_{i}(r) u_{j}(\mathbf{r})\right]=0,
\end{aligned}
$$

for the Bogoliubov transformation to be canonical. The quasiparticles must also be orthogonal to the condensate state, i.e., $\int \mathrm{d} \mathbf{r} \zeta_{0}^{*}(\mathbf{r}) u_{i}(\mathbf{r})=\int \mathrm{d} \mathbf{r} \zeta_{0}(\mathbf{r}) v_{i}(\mathbf{r})=0$. The Bogoliubov equations have the zero-energy solution $\left\{u_{0}(\mathbf{r}), v_{0}(\mathbf{r})\right\}=\left\{\zeta_{0}(\mathbf{r}),-\zeta_{0}^{*}(\mathbf{r})\right\}$, and projection to this homogeneous solution should always be subtracted from the quasiparticle amplitudes.

To calculate the next lowest-order mean fields, i.e., the density of the thermal atoms $\rho(\mathbf{r})=$ $\sum_{i j \neq 0} \zeta_{j}^{*}(\mathbf{r}) \zeta_{i}(\mathbf{r})\left\langle\hat{\alpha}_{j}^{\dagger} \hat{\alpha}_{i}\right\rangle$ and the so-called anomalous average $\kappa(\mathbf{r})=\sum_{i j \neq 0} \zeta_{j}(\mathbf{r}) \zeta_{i}(\mathbf{r})\left\langle\hat{\alpha}_{j} \hat{\alpha}_{i}\right\rangle$, we express the particle operators in terms of the quasiparticle operators, yielding

$$
\begin{aligned}
& \rho(\mathbf{r})=\sum_{i \neq 0}\left\{\left[\left|u_{i}(\mathbf{r})\right|^{2}+\left|v_{i}(\mathbf{r})\right|^{2}\right] n_{i}+\left|v_{i}(\mathbf{r})\right|^{2}\right\}, \\
& \kappa(\mathbf{r})=\sum_{i \neq 0} u_{i}(\mathbf{r}) v_{i}^{*}(\mathbf{r})\left(2 n_{i}+1\right) .
\end{aligned}
$$

In principle, the quasiparticle populations $n_{i}=\left\langle\hat{\beta}_{i}^{\dagger} \hat{\beta}_{i}\right\rangle$ should be calculated from the requirement that the 
canonical partition function $\mathcal{Z}_{\mathrm{c}}=\sum_{\left\{n_{i}\right\}} e^{-\beta E_{i}\left(\left\{n_{i}\right\}\right)} \mathrm{min}-$ imizes the free energy $\mathcal{F}=-k_{\mathrm{B}} T \log \mathcal{Z}_{\mathrm{c}}$. However, to a good approximation [20] one may use the non-interacting quasiparticle gas result $n_{i}=\left(z^{-1} e^{\beta \epsilon_{i}}-1\right)^{-1}$, where the fugacity is calculated from the relation $z=N_{0} /\left(1+N_{0}\right)$.

In calculating the perturbative corrections to the zeroth-order theory corresponding to Eqs. (9) and (10), it is convenient to first calculate the improved condensate wave function $\tilde{\zeta}_{0}(\mathbf{r})$ from the generalized Gross-Pitaevskii (GGP) equation

$$
\begin{aligned}
& -\frac{\hbar^{2}}{2 m} \nabla^{2} \tilde{\zeta}_{0}(\mathbf{r})+V_{\text {trap }}(\mathbf{r}) \tilde{\zeta}_{0}(\mathbf{r})+N_{0} U_{0}\left|\tilde{\zeta}_{0}(\mathbf{r})\right|^{2} \tilde{\zeta}_{0}(\mathbf{r}) \\
& +2 U_{0} \rho(\mathbf{r}) \tilde{\zeta}_{0}(\mathbf{r})+U_{0} \kappa(\mathbf{r}) \tilde{\zeta}_{0}^{*}(\mathbf{r})=\lambda_{\mathrm{g}} \tilde{\zeta}_{0}(\mathbf{r})
\end{aligned}
$$

which is obtained by minimizing $\left\langle\hat{H}_{0}\right\rangle+\left\langle\hat{H}_{2}\right\rangle$. Expressing the terms in the Hamiltonian as

$$
\hat{H}_{i}=\hat{H}_{i}\left[\zeta_{0}\right]+\Delta \hat{H}_{i},
$$

where

$$
\Delta \hat{H}_{i}=\hat{H}_{i}\left[\tilde{\zeta}_{0}\right]-\hat{H}_{i}\left[\zeta_{0}\right],
$$

one finds the perturbative Hamiltonian

$$
\hat{H}_{\text {pert }}=\Delta \hat{H}_{0}+\Delta \hat{H}_{1}+\Delta \hat{H}_{2}+\hat{H}_{3}+\hat{H}_{4},
$$

where the non-quadratic terms $\hat{H}_{3}$ and $\hat{H}_{4}$ are to be calculated using the improved condensate wave function. Note that our notation for $\Delta \hat{H}_{i}$ differs somewhat from that in Refs. $[9,10]$.

The perturbation term $\Delta \hat{H}_{0}$ is just a real number and can be easily taken into account. In addition to it, in first order perturbation theory only the terms $\Delta \hat{H}_{2}$ and $\hat{H}_{4}$ containing even numbers of quasiparticle operators contribute to the energy shift

$$
E_{\text {pert }}(s, 1)=\left\langle s\left|\hat{H}_{\text {pert }}\right| s\right\rangle,
$$

where $|s\rangle$ is a quasiparticle occupation number eigenstate. In second order perturbation theory, one can in fact neglect the terms $\Delta \hat{H}_{2}$ and $\hat{H}_{4}$, because it turns out that their contribution is of the same order as the contribution of the other terms in third order perturbation theory $[9,10]$. Thus, one only needs to calculate

$$
E_{\mathrm{pert}}(s, 2) \approx \sum_{r \neq s} \frac{\left|\left\langle r\left|\Delta \hat{H}_{1}+\hat{H}_{3}\right| s\right\rangle\right|^{2}}{E_{s}-E_{r}} .
$$

The quasiparticle energies are calculated as total energy changes in the system when the corresponding quasiparticle occupation number is increased by one, while the total number of particles is held constant. This yields the corrected excitation energy

$$
E_{p}\left(z^{\prime}\right)=\epsilon_{p}+\Delta E_{4}^{p}+\Delta E_{\text {shape }}^{p}+\Delta E_{\lambda}^{p}+\Delta E_{3}^{p}\left(z^{\prime}\right),
$$

where the $\Delta$-terms are given in Eqs. (A.3)-(A.6) and the complex energy parameter $z^{\prime}$ should not be mixed with the fugacity. Calculating the excitation energies as functions of $z^{\prime}$ yields the dynamics of the excitations in the following way: The time evolution operator $\hat{U}(t)$ of the system may be written in terms of the Fourier transform of the resolvent operator $\hat{G}\left(z^{\prime}\right)=\left(z^{\prime}-\hat{H}\right)^{-1}$ as [21]

$$
\hat{U}(t)=-\frac{\hbar}{\pi} \int_{-\infty}^{\infty} e^{-i \omega t} \operatorname{Im}[\hat{G}(\hbar \omega-i 0)] d \omega .
$$

Let us define the projection of the resolvent to state $p$ as $G_{p}(z)=\langle p|\hat{G}| p\rangle$, which may be approximated to second order as

$$
G_{p}(\omega)=\left[\hbar \omega-E_{p}(\hbar \omega)\right]^{-1} .
$$

Finally, it is seen that the imaginary part of the projected resolvent $F_{p}(\omega-i 0)=\operatorname{Im}\left[G_{p}(\omega-i 0)\right]$ gives the spectral distribution of the mode $p$ and the Fourier transform of $F_{p}(\omega)$ yields its time dependence.

The need to calculate quasiparticle energies as functions of $z^{\prime}$ is naturally related to the fact that one takes into account quasiparticle interactions, though only to the lowest order, and the quasiparticle states are no more energy eigenstates having infinite lifetime. In addition, in computing the quasiparticle energies $z^{\prime}$ must have a small imaginary part acting as a regularizer for the otherwise divergent expressions for second order energy shifts. One may note that setting $z^{\prime}=\epsilon_{p}$ yields the usual Rayleigh-Schrödinger perturbation theory, while the Brillouin-Wigner perturbation theory corresponds to solving the equation $E_{p}\left(z^{\prime}\right)=z^{\prime}$.

In conclusion, the second order theory may be used to calculate the energies and the dynamics of quasiparticles. First the GP equation (9) is solved together with the Bogoliubov equations (10) for a given total particle number $N=N_{0}+N_{\text {ex }}$. Then the GGP equation (14) is solved, after which the spectrum $F_{p}(\omega)$ may be extracted for each excitation $p$ using the energy corrections presented in Eqs. (A.3)-(A.6). In addition, one has to take care of proper ultraviolet renormalization; The quantities $\kappa(\mathbf{r})$ and $\Delta E_{3}^{p}$ are to be replaced by their renormalized values given in Eqs. (A.2) and (A.9) in all calculations.

\section{NUMERICAL METHODS}

We consider a pancake-shaped system in a harmonic potential

$$
V_{\text {trap }}(\mathbf{r})=\frac{1}{2} m \omega_{x}^{2} x^{2}+\frac{1}{2} m \omega_{y}^{2} y^{2}+\frac{1}{2} m \omega_{z}^{2} z^{2},
$$

where the trapping frequencies are $\omega_{r}=\omega_{x}=\omega_{y}$ and $\omega_{z}$, with $\omega_{z} \gg \omega_{r}$. For a sufficiently strong trapping potential in the $z$-direction, the condensate wave function and the thermodynamically relevant quasiparticle amplitudes can be approximated to be in cylindrical coordinates $(r, \theta, z)$ of the factorized form

$$
\zeta_{0}(\mathbf{r})=\zeta_{0}(r) \sigma(z) e^{i m \theta},
$$


and

$$
\begin{aligned}
& u(\mathbf{r})=u(r) \sigma(z) e^{i\left(q_{\theta}+m\right) \theta}, \\
& v(\mathbf{r})=v(r) \sigma(z) e^{i\left(-q_{\theta}+m\right) \theta},
\end{aligned}
$$

where $\sigma(z)=e^{-z^{2} /\left(2 a_{z}^{2}\right)} / \sqrt{a_{z} \pi^{1 / 2}}$ is a Gaussian profile and $a_{i}=\sqrt{\hbar / m \omega_{i}}$ are the harmonic oscillator lengths of the trap. In the following, we consider only the case $m=0$ of an irrotational condensate. Using Eqs. (23), (24) and (25), the Gross-Pitaevskii and Bogoliubov equations reduce to equations of the radial coordinate only. The $z$-dependence of the GP, the GGP and the Bogoliubov equations is reduced explicitly by multiplying them with $\sigma(z)$ and integrating over $z$. This results in equations similar to the original ones except that the interaction strength $U_{0}=4 \pi \hbar^{2} a / m$ is replaced with its quasi two-dimensional version $U_{0}^{2 \mathrm{D}}=2 \sqrt{2 \pi} \hbar \omega_{z} a_{z} a$ and the chemical potential is shifted by $\hbar \omega_{z} / 2$. We use $a_{r}, 1 / \omega_{r}$ and $\hbar \omega_{r}$ as units of length, time and energy, respectively. In these units the dimensionless interaction strength becomes $U_{0}^{2 \mathrm{D}}=2 \sqrt{2 \pi} a / a_{z}$. A peculiarity of the reduced equations is that they are independent of the trapping frequency $\omega_{r}$, and hence our results apply for all $\omega_{r}$, provided that $\omega_{z} \gg \omega_{r}$.

In numerical calculations, we use spatial discretization and finite-difference methods. The ground state solutions of the non-linear GP and GGP equations are found by a norm-conserving imaginary time integration method based on the Crank-Nicholson scheme. The computation speed is enhanced by using a multigrid method, in which the grid is made gradually denser during the computation. On the other hand, in spatial discretization the Bogoliubov eigenvalue equation becomes a matrix eigenvalue equation, with the coefficient matrix having a narrow band. The eigenvalues of this matrix are found by implicitly restarted Arnoldi method implemented in the numerical library ARPACK. The quasiparticle amplitudes are calculated from the Bogoliubov equations up to some cut-off energy $E_{\text {cut }}$, above which a semiclassical approximation [22] is used for calculating their contribution to mean-field potentials. The use of the semiclassical approximation enhances the convergence of the results as a function of $E_{\text {cut }}$.

The anomalous average and the energy shifts $\Delta E_{3}^{p}$ contain ultraviolet divergences due to the interaction contact potential approximation, and they have to be renormalized. The renormalization scheme presented in the Appendix shows that the divergent part of the anomalous average is proportional to $\tilde{\zeta}_{0}^{2}(\mathbf{r})$. Using this information we determine the value of the interaction correction $\Delta U_{0}\left(E_{\text {cut }}\right)$ due to the excitations below $E_{\text {cut }}$. This coefficient also suffices to determine the proper renormalization subtraction for the energy shift $\Delta E_{3}^{p}$. As an approximation to this proper renormalization, the ultraviolet divergences can also be removed by neglecting the zero-temperature parts of the terms $\kappa(\mathbf{r})$ and $\Delta E_{3}^{p}$. For dilute gases this should be a good approximation [10]. This simpler renormalization method is also computa- tionally much faster, since the summations in the energy correction $\Delta E_{3}^{p}$ converge and many terms may be neglected within computational accuracy. In the more accurate renormalization scheme that we have used, the summations diverge and hence all the terms up to $E_{\text {cut }}$ have to be taken into account.

In calculating the spectral distributions $F_{p}(\omega-i \gamma)$, we have to use a finite imaginary part $\gamma$ to avoid divergences in Eq. (A.6). The value of $\gamma$ is estimated for each excitation and temperature separately to be small enough for not to affect the mean value of the spectral distribution nor its Fourier transform in the regime we have presented it. In a finite system, the spectral distributions consist of discrete Lorentzian peaks with widths proportional to $\gamma$. Thus, when calculating the spectral density, the smaller the regulator $\gamma$ is, the finer grid for the real part $\omega$ must be used. Together with the double summation in Eq. (A.6), this can increase the computational cost of the spectrum to be orders of magnitude larger than the cost needed in solving the excitations from the Bogoliubov equations. To make the computation of the spectrum more efficient, the terms $A_{i j k}$ and $B_{i j k}$ in Eq. (A.6) are calculated only once and stored in memory. In addition, a comparable speedup is achieved by regrouping the summation terms according to their behaviour as functions of $\omega$ : for slowly varying terms, one can use much sparser $\omega$-discretization.

We note that in the end we take the limit $\gamma \rightarrow 0+$, as was suggested in Ref. [10]. However, in Refs. [2, 16, 17] a finite value of the order $\gamma \sim 10^{-2} \omega_{r}$ was used, motivated by the finite experimental observation time, and in Ref. [18] the value of $\gamma$ was taken to be $5 \times 10^{-3} \omega_{r}$. However, the inclusion of a finite $\gamma$ is only one way to model the finite observation time. Since the finite value of the regulator $\gamma$ has also an unphysical effect of shifting the excitation energies, it might not be the best way to model the restricted observation time.

\section{EXCITATION SPECTRA}

In this section, we present and analyze the results for the mean energies of excitations as functions of temperature. We model a pancake-shaped cloud consisting of $N=2000{ }^{23} \mathrm{Na}$ atoms trapped in the tight direction with the trapping frequency $\omega_{z}=2 \pi \times 350 \mathrm{~Hz}$. As pointed out in Sec. III, the radial trapping frequency $\omega_{r}=\omega_{x}=\omega_{y}$ may be chosen freely with only the constraint $\omega_{z} \gg \omega_{r}$. These parameters are chosen for convenience to coincide with the ones used in Ref. [23], in which the excitation energies were calculated from the self-consistent HFBPopov theory. Previously, energies of a few modes for spherically symmetric systems [17] and condensates containing a vortex line [18] have been computed within this second order theory.

Figure 1 shows the zeroth order energies of the lowest energy excitations at zero temperature. Within the accuracy of the figure, the zeroth order Bogoliubov energies 


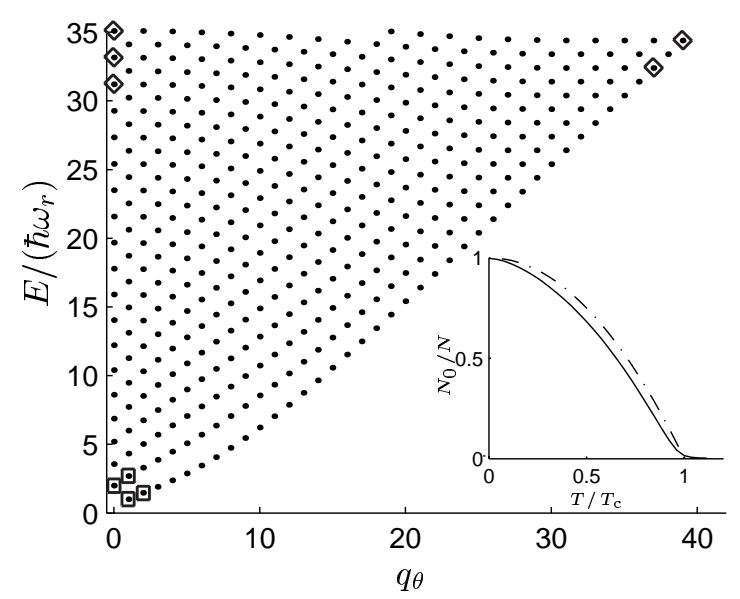

FIG. 1: Excitation energies of the lowest part of the spectrum at zero temperature. The energies of excitations marked with squares and diamonds are presented as functions of temperature in Figs. 2 and 3, respectively. The inset shows the condensate fraction as function of temperature (solid line), and the exact result $1-\left(T / T_{\mathrm{c}}\right)^{2}$ for the non-interacting system (dashdot line).

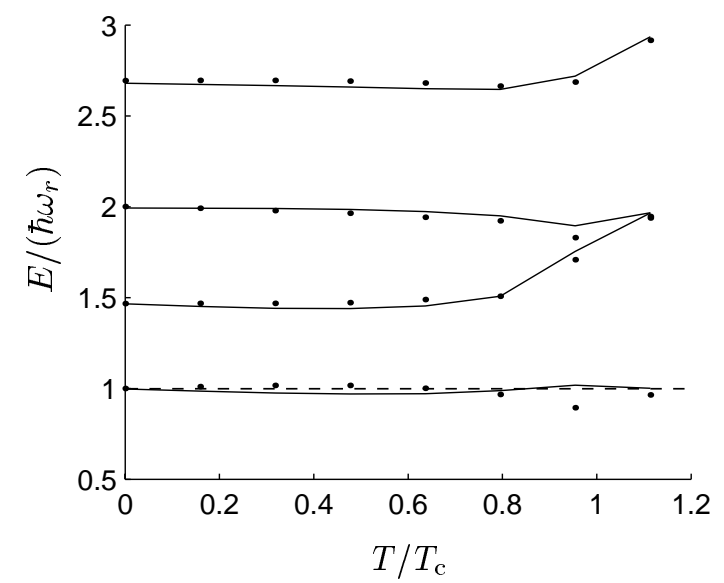

FIG. 2: Temperature dependence of the mean energies of the modes marked with squares in Fig. 1. Dots correspond to the second order theory, and solid lines to the HFB-Popov theory. The dashed line indicates the exact energy $\hbar \omega_{r}$ of the Kohn modes.

coincide with those of the full second order theory. Since the condensate is irrotational, the spectrum is symmetric with respect to inversion $q_{\theta} \rightarrow-q_{\theta}$, and thus only excitations with non-negative angular momenta are shown. The inset presents the condensate fraction as a function of the temperature (solid line) compared with the noninteracting gas result $N_{0}^{\prime}(T) / N=1-\left(T / T_{\mathrm{c}}\right)^{2}$ (dashdot line). We identify the condensation temperature $T_{\mathrm{c}}$ as the point where the condensate fraction obtains its maximum second derivative with respect to the temperature. The theory is probably not reliable above or in the vicinity $T_{\mathrm{c}}$, although we present its predictions also in this regime.

The energies of the modes marked with squares and diamonds in Fig. 1 are presented as functions of temperature in Figs. 2 and 3, respectively. The mean values of the spectral distributions of the excitations within the second order theory are shown, in addition to the corresponding HFB-Popov results that were obtained by neglecting the anomalous average, the second order corrections and the terms in the second line of Eq. (A.3). For the low-lying modes, some of the second order energies are observed to cross the Popov results as seen in Fig. 2. On the other hand, Fig. 3 shows that the second order theory for the higher lying modes yields systematically lower excitation energies than the Popov theory. The Popov results seen in Fig. 2 are consistent with the energies calculated in Ref. [23].

In Fig. 2, the dashed line corresponds to the energy $\hbar \omega_{r}$ of the exact center of mass oscillation modes, the Kohn modes. According to the generalization [24, 25] of Kohn's theorem [26], a system of harmonically trapped interacting particles in any eigenstate of the Hamiltonian has an eigenstate with the amount $\hbar \omega_{i}$ higher energy, i.e., the exact diagonalization of the Hamiltonian should yield a spectrum with the eigenenergy $\hbar \omega_{i}$. The Bogoliubov theory, in which the thermal gas component is neglected, implies Kohn modes to have this exact energy. In the higher order theories, the dynamics of the thermal gas and its interaction with the condensate have to be taken into account accurately to obtain results in agreement with the Kohn theorem. Figure 2 shows that within the second order theory the energy of the Kohn mode is very close to $\hbar \omega_{r}$ for temperatures $T<0.8 T_{\mathrm{c}}$. Taking into account perturbation theory terms beyond the second order should yield energies even closer to the exact result.

The lowest mode with vanishing angular momentum is the breathing mode corresponding to uniform scaling oscillations of the condensate. In the case of a twodimensional harmonically trapped gas interacting via the contact potential, it has been shown using the scaling symmetry of the Hamiltonian that there exists a state that has energy $2 \hbar \omega_{r}$ in excess to the ground state [27]. This excitation is identified with the breathing mode. The Bogoliubov theory yields exactly the energy $2 \hbar \omega_{r}$ for the breathing mode, while the Popov and the second order theories do not, as can be seen in Fig. 2. Since the interaction potential has to be renormalized and hence deviates from that used in Ref. [27] for modes with high energy, the applicability of the exact result is somewhat questionable at high temperatures, where the physics is not determined by the low-lying modes alone. It is shown in Fig. 2 that the energy of the breathing mode is lower than $2 \hbar \omega_{r}$ and the deviation from $2 \hbar \omega_{r}$ increases with the temperature. 


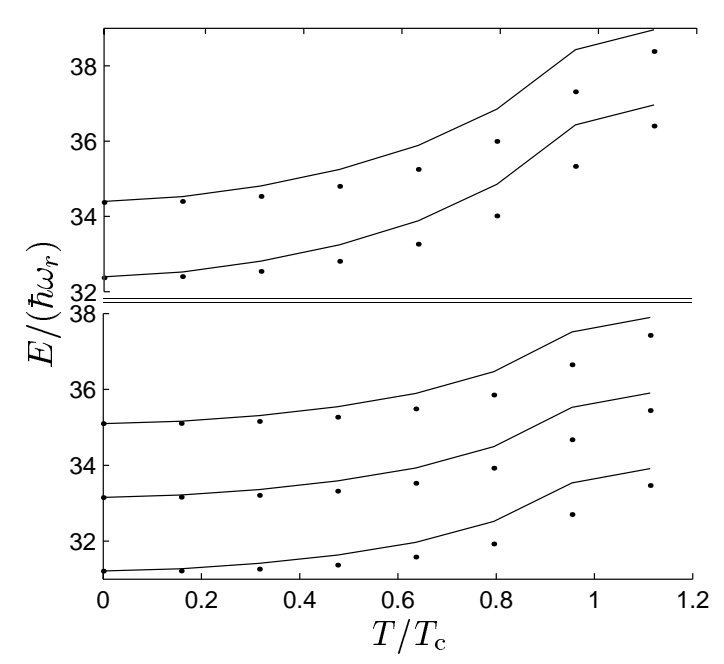

FIG. 3: Temperature dependence of the mean energies of the modes marked with diamonds in Fig. 1 within second order theory (dots) compared with the HFB-Popov results (solid lines). The energies in the lower block correspond to excitations with vanishing angular momentum and the upper block to excitations with $q_{\theta} \approx 40$.

\section{DECAY OF THE EXCITATIONS}

In experiments and theoretical studies, the decay of an excitation is commonly characterized only by the damping rate related to the exponential decay of the oscillation amplitude. For infinite systems, the excitation spectrum is continuous and the spectral distributions $F_{p}(\omega)$ of the excitations are Lorentzian peaks, implying indeed an exponential decay of the mode oscillations. The mean value of the Lorentzian gives the mode frequency and its width the damping rate. However, for trapped, finite systems the spectrum is discrete and the spectral distributions generally have more complicated forms. Especially, the dynamics implied by these distributions can be more complicated than just the simple exponential decay. From the computed spectral distributions of the oscillations, we have studied the validity of the exponential decay approximation for the finite system under question.

In fact, the computed spectral distributions consist of discrete peaks, as noted also previously $[17,18]$, and the form of the distributions is typically far from a simple Lorentzian profile. This seems to imply the excitation amplitudes to have a complicated modulation in time. The extreme case of this modulation is the collapse and revival of the corresponding excitation amplitude. This phenomenon can be seen for the breathing mode at zero temperature. Figure 4(a) displays the computed spectral distribution for the breathing mode, which consists of two large, well separated peaks. This implies strong beating behaviour in the mode amplitude, seen in Fig. 4(b), in which the amplitude of the oscillation collapses in time $t=45 / \omega_{r}$, but revives as time elapses. In fact, the am-
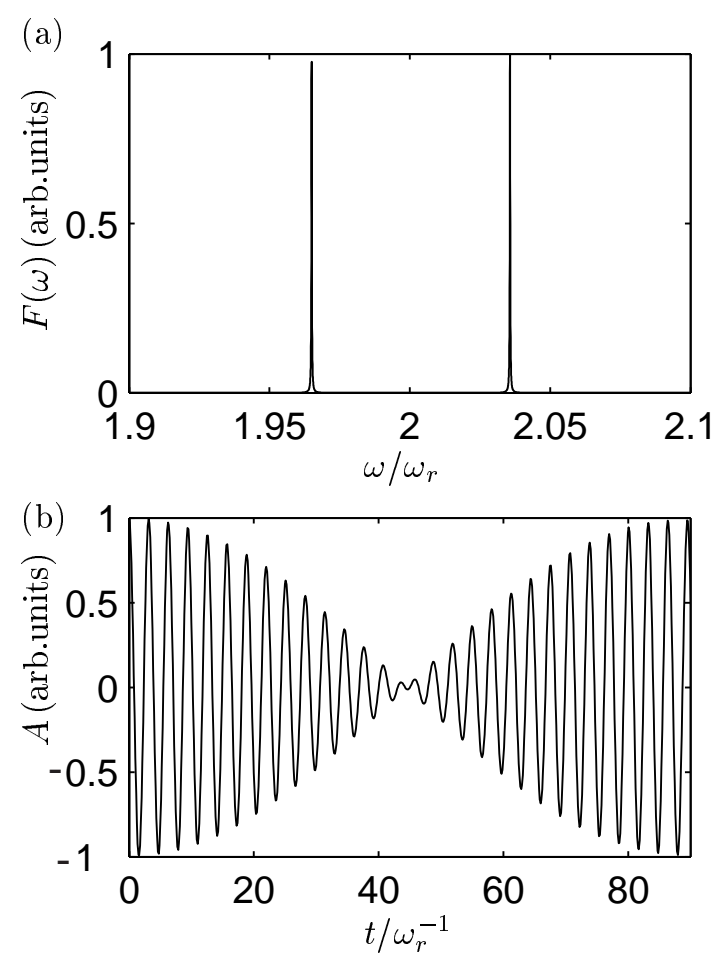

FIG. 4: (a) Spectral distribution $F(\omega)$ of the breathing mode (the lowest mode with $q_{\theta}=0$ ) at zero temperature and (b) its Fourier transform.

plitude has a beating behaviour with a base frequency given by the mean value of the peaks in Fig. 4(a), and a beating frequency inversely proportional to the distance between the peaks.

The two peaks in Fig. 4(a) are due to a Beliaev process [28] resonance, in which a breathing mode quasiparticle with Bogoliubov energy $2 \hbar \omega_{r}$ decays into two Kohn mode quanta with opposite angular momenta and energy $\hbar \omega_{r}$. Owing to the temperature independent terms in Eq. (A.6), the Beliaev process may take place even at zero temperature. For the simpler, approximate renormalization scheme in which the temperature independent terms in the anomalous average and the energy correction $\Delta E_{3}$ are completely neglected, this Beliaev process cannot occur at zero temperature, and the spectral distribution consists of only a single peak. For the Kohn mode, there are no modes into which it could decay via Beliaev processes and hence the oscillation amplitude of the center of mass is constant in time at zero temperature. At finite temperatures, the resonant Landau process, the inverse of the Beliaev process, splits the spectral distribution of the Kohn modes.

The non-trivial spectral distributions of the breathing mode and the Kohn modes seem to contradict the results for the exact energies of these modes discussed above. This is partly due to the accidental strong resonant Beliaev and Landau processes which probably weaken the accuracy of perturbation theory. If the processes, in which the quasiparticles decay into Kohn or breathing modes, 

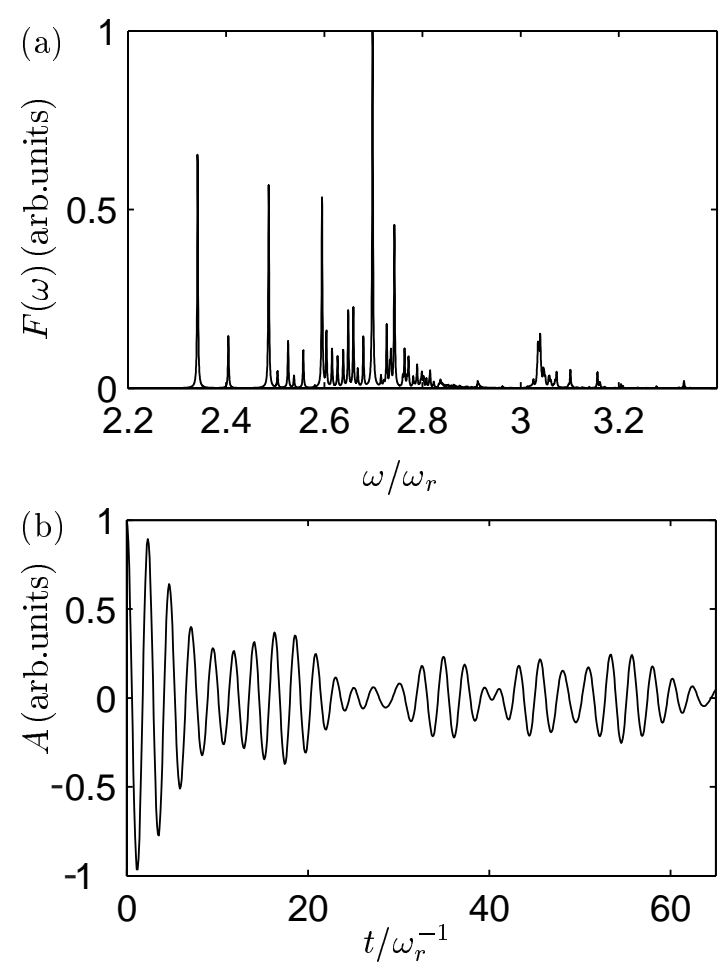

FIG. 5: (a) Spectral distribution $F(\omega)$ of the second lowest mode with $q_{\theta}=1$ and (b) its Fourier transform at the temperature $T=0.64 T_{\mathrm{c}}$.

are neglected by hand as in Ref. [29], the effects of the resonances are removed and the spectral distributions of these modes become narrower. However, it is not evident that this procedure yields more accurate mean energies. In conclusion, a pure collapse and revival of the breathing mode is probably only an artefact of the second order theory - it would be interesting to investigate whether higher order calculations would improve the situation in this respect.

The spectral distribution and the dynamics of the second lowest mode with $q_{\theta}=1$ at the temperature $T=0.64 T_{\mathrm{c}}$ is shown in Fig. 5. The distribution is obviously far from Lorentzian form, consisting of several asymmetrically separated peaks, and the dynamics is more complicated than the zero temperature result for the breathing mode presented in Fig. 4(b). The collapse and revival behaviour is clearly seen, although it is weaker than in Fig. 4(b). Our calculations have also showed collapse and ravival of many other elementary excitations.

In Ref. [30], the Beliaev decay has been reported to be observed in the case of the scissors mode which corresponds to scissors-like density fluctuation of the condensate. In the experiment, the trapping frequency ratio $\omega_{z} / \omega_{x}$ was adjusted such that the energy $E_{\mathrm{xz}}$ of the scissors mode in the $x z$-plane was twice the energy $E_{\mathrm{xy}}$ in the $x y$-plane. The amplitude of the oscillation is shown in Fig. 3(c) of Ref. [30], and it was observed that the am- plitude of the mode decreases and increases in time. It was also observed that the strength of this phenomenon was peaked into the position of the Beliaev resonance as a function of the trap asymmetry ratio $\omega_{x} / \omega_{y}$, implying that the Beliaev process between these modes is responsible for this effect.

Within the second order formalism, one can interpret this phenomenon in the following way: Provided that the numerator in the term corresponding to the Beliaev decay of the scissors mode does not vanish in Eq. (A.6) and other processes are not important, the second order theory yields a spectral distribution proportional to

$$
F_{\mathrm{sc}}(\omega)=\operatorname{Im}\left[1 /\left(\hbar \omega-0 i-E_{\mathrm{xz}}+A /\left(\hbar \omega-0 i-E_{\mathrm{xz}}+E_{\mathrm{ofr}}\right)\right)\right],
$$

where $A$ is the amplitude of the second order correction and $E_{\text {ofr }}$ is the energy indicating how much the Beliaev process is off-resonant. If $E_{\mathrm{ofr}}=0$ the distribution $F_{\mathrm{sc}}(\omega)$ consists of two peaks whose distance is determined by $A$, and the dynamics of the mode corresponds a pure collapse and revival as shown in Fig. 4 in the case of the breathing mode. With increasing $E_{\text {ofr }}$, one of the two peaks becomes smaller and the peaks are shifted in a such way that their mean value is kept at $E_{\mathrm{xz}}$. This corresponds to an oscillation in which the amplitude does not vanish completely at any moment, and ultimately when $E_{\text {ofr }} \rightarrow \infty$ it remains constant, which qualitatively explains the observation in the experiments. It is possible that due the parity of the scissors modes the second order amplitude vanishes. However, the higher order corrections may still have non-vanishing amplitudes, resulting in qualitatively same kind of effect.

\section{CONCLUSIONS}

We used the gapless second order theory developed in Refs. $[9,10]$ to calculate the excitation energies and dynamics of the collective excitations for a partially condensed, harmonically trapped quasi $2 \mathrm{D}$ bosonic gas. The results satisfy the Kohn theorem quite accurately for temperatures $T<0.8 T_{\mathrm{c}}$. The energies of the HFB-Popov and the second order theory crossed as functions of temperature for some of the low-lying modes, while the second order theory systematically yields smaller energies for the higher lying modes. The first experimental observations of the Beliaev damping were discussed within the second order theory and it was found that this theory qualitatively accounts for the observations.

The computed spectral densities also imply collapse and revival of many elementary excitations. The zero temperature spectral distribution of the breathing mode is characterized by two large, well-separated peaks and the oscillation amplitude consequently displays strong collapse and revival behaviour within the second order theory. This is due to the resonant Beliaev process, in which one quasiparticle in the breathing mode decays into two quasiparticles in the Kohn modes with opposite angular momenta. The result seems to contradict exact 
analytical results for the breathing mode energy [27], and is probably due to weak convergence of the perturbation theory for this mode. The calculations can in principle be extended to higher order, but this soon results in overwhelming computational difficulties. It would also be interesting to upgrade the calculations to be selfconsistent $[9,10]$, such that the perturbative energy corrections are inserted into the eigenvalue equations, which are then solved iteratively.

\section{Acknowledgments}

The Academy of Finland is appreciated for financial support through a Research Grant in Theoretical Materials Physics (no 201710) and CSC-Scientific Computing Ltd (Espoo, Finland) for computational resources. M. Möttönen acknowledges The Foundation of Technology (Helsinki, Finland) and The Finnish Cultural Foundation for financial support. J. J. Vartiainen is appreciated for discussions.

\section{APPENDIX: ULTRAVIOLET RENORMALIZATION}

Since the low-energy effective contact potential approximation for the interactions between the particles is not valid at high energies, the bare anomalous average $\kappa(\mathbf{r})$ is ultraviolet divergent. To remove this divergence in a proper way, following Ref. [16], the interaction strength $U_{0}$ in GGP equation (14) must be replaced by $U_{0}+\Delta U_{0}$, where

$$
\Delta U_{0}=U_{0}^{2} \int \frac{\mathrm{d} \mathbf{k}}{(2 \pi)^{3}} \frac{m}{\hbar^{2} k^{2}}
$$

The term $\Delta U_{0}$ is divergent, and cancels the divergence of the anomalous average. Combining the divergent terms, one obtains the renormalized anomalous average

$$
\kappa^{\mathrm{R}}(\mathbf{r})=\kappa(\mathbf{r})+N_{0} \frac{\Delta U_{0}}{U_{0}} \tilde{\zeta}_{0}^{2}(\mathbf{r})
$$

which is finite. The GGP equation is now properly renormalized provided that the anomalous average is replaced by the renormalized one.

The perturbation Hamiltonian given in Eq. (17) is used to calculate the total energy of the system $E\left(N_{0}, n_{1}, n_{2}, \ldots\right)$ up to second order in perturbation theory for the given quasiparticle distribution $\left\{n_{i}\right\}$. The excitation energies are obtained as energy differences $E_{p}=$ $E\left(N_{0}-\Delta N_{p}, n_{1}, n_{2}, \ldots, n_{p}+1, \ldots\right)-E\left(N_{0}, n_{1}, n_{2}, \ldots\right)$, where $\Delta N_{p}=\int \mathrm{d} \mathbf{r}\left[\left|u_{i}(\mathbf{r})\right|^{2}+\left|v_{i}(\mathbf{r})\right|^{2}\right]$ is the amount of particles transferred to the mode $p$. The energy correc- tion terms appearing in Eq. (20) are given by

$$
\begin{aligned}
\Delta E_{4}^{p} & =U_{0} \int \mathrm{d} \mathbf{r}\left[2 \rho(\mathbf{r}) \Delta \rho_{p}(\mathbf{r})\right. \\
+ & \operatorname{Re}\left[\kappa^{*}(\mathbf{r}) \Delta \kappa(\mathbf{r})\right]-\frac{n_{p}+1}{2}\left\{2 \Delta \rho_{p}^{2}(\mathbf{r})\right. \\
+ & \left.\left.\operatorname{Re}\left[\Delta \kappa^{*}(\mathbf{r}) \Delta \kappa(\mathbf{r})\right]\right\}\right] \\
\Delta E_{\text {shape }}^{p}= & N_{0} U_{0} \int \mathrm{d} \mathbf{r} \\
\times & \operatorname{Re}\left\{\left[\tilde{\zeta}_{0}^{2}(\mathbf{r})-\zeta_{0}^{2}(\mathbf{r})\right] \Delta \kappa_{p}(\mathbf{r})\right\} \\
+ & 2 N_{0} U_{0} \int \mathrm{d} \mathbf{r}\left[\tilde{\zeta}_{0}^{2}(\mathbf{r})-\zeta_{0}^{2}(\mathbf{r})\right] \Delta \rho_{p}(\mathbf{r}) \\
+ & 2 N_{0} U_{0} \int \mathrm{d} \mathbf{r} \zeta_{0}^{3}(\mathbf{r})\left[u_{p}(\mathbf{r})+v_{p}(\mathbf{r})\right] \\
\times & \int \mathrm{d} \mathbf{r}\left[\zeta_{0}(\mathbf{r})-\tilde{\zeta}_{0}(\mathbf{r})\right]\left[u_{p}(\mathbf{r})+v_{p}(\mathbf{r})\right], \\
\Delta & E_{\lambda}^{p}=\left(\lambda-\lambda_{\mathrm{g}}\right) \int \mathrm{d} \mathbf{r} \Delta \rho_{p}(\mathbf{r}), \\
\Delta E_{3}^{p}\left(z^{\prime}\right)= & -\sum_{j i \neq 0}^{\prime}\left[\frac{2\left|A_{p i j}\right|^{2}}{z^{\prime}+\epsilon_{i}+\epsilon_{j}}\right. \\
& \left.+\frac{2\left|B_{p i j}\right|^{2}}{z^{\prime}-\epsilon_{i}-\epsilon_{j}}\right]\left(1+n_{i}+n_{j}\right) \\
& +\sum_{i j \neq 0}^{\prime}\left[\frac{4\left|B_{i j p}\right|^{2}}{z^{\prime}-\epsilon_{i}+\epsilon_{j}}\left(n_{i}-n_{j}\right)\right] \\
& +\sum_{i \neq 0}^{\prime}\left[\frac{4\left|B_{p i p}\right|^{2}}{\epsilon_{i}}\left(2 n_{p}+1\right)\right. \\
& \left.-2 n_{p} \frac{\left|B_{p p i}\right|^{2}}{2 z^{\prime}-\epsilon_{i}}-2 n_{p} \frac{\left|A_{i p p}\right|^{2}}{2 z^{\prime}+\epsilon_{i}}\right],
\end{aligned}
$$

where in the primed summations one excludes the terms in which all the summation indices are equal to $p$. These diagonal terms are negligible in the current calculations. The contribution to the density of the thermal gas and the anomalous average due to the mode $p$ are defined as $\Delta \rho_{p}(\mathbf{r})=\left|v_{p}(\mathbf{r})\right|^{2}+\left|u_{p}(\mathbf{r})\right|^{2}$ and $\Delta \kappa_{p}(\mathbf{r})=u_{p}(\mathbf{r}) v_{p}^{*}(\mathbf{r})$. Moreover, we have replaced the energy $\epsilon_{p}$ with a complex variable $z^{\prime}$. The second order matrix elements are written as

$$
\begin{aligned}
A_{i j k} & =\sqrt{N_{0}} U_{0} \int \mathrm{d} \mathbf{r}\left\{\zeta _ { 0 } ( \mathbf { r } ) \left[v_{i}(\mathbf{r}) u_{j}(\mathbf{r}) u_{k}(\mathbf{r})\right.\right. \\
& \left.+u_{i}(\mathbf{r}) v_{j}(\mathbf{r}) u_{k}(\mathbf{r})+u_{i}(\mathbf{r}) u_{j}(\mathbf{r}) v_{k}(\mathbf{r})\right] \\
& +\zeta_{0}^{*}(\mathbf{r})\left[u_{i}(\mathbf{r}) v_{j}(\mathbf{r}) v_{k}(\mathbf{r})\right. \\
& \left.\left.+v_{i}(\mathbf{r}) u_{j}(\mathbf{r}) v_{k}(\mathbf{r})+v_{i}(\mathbf{r}) v_{j}(\mathbf{r}) u_{k}(\mathbf{r})\right]\right\}, \\
B_{i j k} & =\sqrt{N_{0}} U_{0} \int \mathrm{d} \mathbf{r}\left\{\zeta _ { 0 } ^ { * } ( \mathbf { r } ) \left[u_{i}^{*}(\mathbf{r}) u_{j}(\mathbf{r}) u_{k}(\mathbf{r})\right.\right. \\
& \left.+v_{i}^{*}(\mathbf{r}) v_{j}(\mathbf{r}) u_{k}(\mathbf{r})+v_{i}^{*}(\mathbf{r}) u_{j}(\mathbf{r}) v_{k}(\mathbf{r})\right] \\
& +\zeta_{0}(\mathbf{r})\left[u_{i}^{*}(\mathbf{r}) u_{j}(\mathbf{r}) v_{k}(\mathbf{r})\right. \\
& \left.\left.+u_{i}^{*}(\mathbf{r}) v_{j}(\mathbf{r}) u_{k}(\mathbf{r})+v_{i}^{*}(\mathbf{r}) v_{j}(\mathbf{r}) v_{k}(\mathbf{r})\right]\right\} .
\end{aligned}
$$


The energy correction $\Delta E_{3}^{p}$ in Eq. (A.6) is ultraviolet divergent. However, the renormalization of the anomalous average implies that the bare second order correction $E_{3}^{p}(z)$ is to be replaced by the renormalized one

$$
\begin{aligned}
\Delta E_{3 \mathrm{R}}^{p}\left(z^{\prime}\right) & =E_{3}^{p}\left(z^{\prime}\right)+2 N_{0} \Delta U_{0} \\
& \times \int \mathrm{d} \mathbf{r}\left|\zeta_{0}(\mathbf{r})\right|^{2}\left(\left|u_{p}(\mathbf{r})\right|^{2}+\left|v_{p}(\mathbf{r})\right|^{2}\right)
\end{aligned}
$$

[1] R. J. Dodd, M. Edwards, C. W. Clark, and K. Burnett, Phys. Rev. A 57, R32 (1998).

[2] S. A. Morgan, M. Rusch, D. A. W. Hutchinson, and K. Burnett, Phys. Rev. Lett. 91, 250403 (2003).

[3] A. Minguzzi and M. P. Tosi, J. Phys.: Condens. Matter 9, 10211 (1997).

[4] G. Bene and P. Szépfalusy, Phys. Rev. A 58, R3391 (1998).

[5] P. O. Fedichev and G. V. Shlyapnikov, Phys. Rev. A 58, 3146 (1998).

[6] M. Rusch and K. Burnett, Phys. Rev. A 59, 3851 (1999).

[7] J. Reidl, A. Csordás, R. Graham, and P. Szépfalusy Phys. Rev. A 61, 043606 (2000).

[8] S. Giorgini, Phys. Rev. A 61, 063615 (2000).

[9] S. A. Morgan, J. Phys. B 33, 3825 (2000).

[10] S. A. Morgan, PhD Thesis, University of Oxford (1999).

[11] E. Zaremba, A. Griffin, and T. Nikuni, Phys. Rev. A 57, 4695 (1998).

[12] T. Nikuni, E. Zaremba, and A. Griffin, Phys. Rev. Lett. 83, 10 (1999).

[13] E. Zaremba, T. Nikuni, and A. Griffin, J. Low Temp. Phys. 116, 277 (1999).

[14] R. Walser, J. Williams, J. Cooper, and M. Holland, Phys. Rev. A 59, 3878 (1999).

[15] N. P. Proukakis, J. Phys. B 34, 4737 (2001).

[16] S. A. Morgan, Phys. Rev. A 69, 023609 (2004).
[17] M. Rusch, S. A. Morgan, D. A. W. Hutchinson, and K. Burnett, Phys. Rev. Lett. 85, 4844 (2000).

[18] T. Mizushima, M. Ichioka, and K. Machida, Phys. Rev. Lett. 90, 180401 (2003).

[19] L. P. Pitaevskii, Phys. Lett. A 229, 406 (1997).

[20] T. Bergeman, D.L. Feder, N.L. Balazs, and B.I. Schneider, Phys. Rev. A 61, 063605 (2000).

[21] C. Cohen-Tannoudji, J. Dupont-Roc, and G. Grynberg, Atom-Photon Interactions (Wiley, New York, 1992).

[22] J. Reidl, A. Csordás, R. Graham, and P. Szépfalusy Phys. Rev. A 59, 3816 (1999).

[23] C. Gies, B. P. van Zyl, S. A. Morgan, and D. A. W. Hutchinson Phys. Rev. A 69, 023616 (2004).

[24] J. F. Dobson, Phys. Rev. Lett. 73, 2244 (1994).

[25] A. L. Fetter and D. Rokhsar, Phys. Rev. A 57, 1191 (1998).

[26] W. Kohn, Phys. Rev. 123, 1242 (1961).

[27] L. P. Pitaevskii and A. Rosch, Phys. Rev. A 55, R853 (1997).

[28] S. T. Beliaev, Zh. Éksp. Teor. Fiz. 7, 469 (1958) [Sov. Phys. JETP 34, 322 (1958)].

[29] M. Guilleumas and L. P. Pitaevskii, Phys. Rev. A 61, 013602 (1999).

[30] E. Hodby, O. M. Maragó, G. Hechenblaikner, and C. J. Foot, Phys. Rev. Lett. 86, 2196 (2001). 